\title{
La alimentación del adolescente: necesidad imperiosa de actuar de forma inmediata
}

\section{Adolescent nutrition: an urgent need to act immediately}

\author{
A. Martín ${ }^{1,2}$, J.A. Martínez ${ }^{1,2}$
}

Algunos adolescentes exhiben hoy en día patologías propias del adulto como la diabetes tipo 2, hígado graso, o apnea del sueño. Nuestro país presenta altas cifras de obesidad en la etapa infantojuvenil. La prevalencia de sobrepeso y obesidad es del $29 \%$ en niños y $26,5 \%$ en niñas de 2-17 años según la Encuesta de Salud 2011-12 y uno de cada 3 adolescentes tiene exceso de peso ${ }^{1}$.

En el sujeto con sobrepeso u obeso aparece una constelación de factores de riesgo cardiovascular que constituyen el llamado "síndrome metabólico". Una de ellas es la obesidad central que empieza ya en la adolescencia. En España hay un 14,3\% de adolescentes de 12 a 17 años con obesidad abdominal ${ }^{2}$ lo que se asocia a un mayor riesgo cardiometabólico. Además, los adolescentes obesos pueden presentar más frecuentemente: trastorno por déficit de atención con hiperactividad, trastornos de la conducta, depresión, problemas de aprendizaje, retraso en el desarrollo, problemas de articulaciones, alergias, dolor de cabeza e infecciones de óticas ${ }^{3}$. Estas alteraciones pueden repercutir en la calidad de vida e incluso en la longevidad.

El peso de los padres es el factor determinante para la epidemia de obesidad infantil incluso con independencia del nivel socioeconómico. Los niños con al menos un padre obeso tiene cuatro veces mayor probabilidad de llegar a ser ellos mismos obesos ${ }^{4}$.

La alimentación en la adolescencia tiene diversas finalidades aparte de cubrir las necesidades energéticas, plásticas y reguladoras que permitan el crecimiento y desarrollo óptimo del organismo y de evitar carencias y desequilibrios entre nutrientes. Es una etapa clave en la que deben promover hábitos alimentarios correctos que ayuden en la prevención de enfermedades crónicas en la vida adulta. Como es un periodo de cambio y desarrollo supone un importante reto para los

1. Departamento de Nutrición, Ciencias de la Alimentación y Fisiología, Universidad de Navarra, Pamplona.

2. Centro de Investigación Biomédica en Red de Fisiopatología de la Obesidad y Nutrición (CIBERobn), Fisiopatología de la Obesidad y Nutrición, Instituto de Salud Carlos III, Madrid.
Corresponencia:

A. Martí del Moral
Departamento de Nutrición
Ciencias de la Alimentación y Fisiología
Universidad de Navarra
C/ Irunlarrea, s/n
31008 Pamplona. Navarra
E-mail. amarti@unav.es

A. Martí del Moral

Departamento de Nutrición

Ciencias de la Alimentación y Fisiología

Universidad de Navarra

31008 Pamplona. Navarra

E-mail. amarti@unav.es 
educadores y profesionales sanitarios la supervisión de los objetivos ya que los adolescentes van aumentando su grado de autonomía ${ }^{5-7}$. Necesitan un fuerte apoyo familiar, su personalidad se va configurando en esas etapas, aunque la mayor influencia la ejercen sus compañeros y grupos de amigos, sin contar con la influencia de los mass media y de las TICS. Así, la adolescencia se considera un período de la vida especialmente vulnerable desde el punto de vista de la nutrición, donde con frecuencia pueden aparecer trastornos de la conducta alimentaria (anorexia, bulimia, etc.).

Por otro lado, los requerimientos nutricionales durante la adolescencia dependen del sexo y de factores individuales como el ritmo de crecimiento y la maduración, junto con el grado de actividad física. El trabajo de Palenzuela y $\mathrm{col}^{8}$, que se publica en este número de la revista, ofrece los resultados sobre las características de la alimentación de escolares de la provincia de Córdoba, un total de 531 varones y $474 \mathrm{mu}-$ jeres de 11,45 años. Los datos se recogieron mediante un cuestionario de frecuencia de consumo semanal (5 opciones categorizadas) de diferentes tipos de alimentos. Este trabajo considera también la profesión de los padres, donde predominan los trabajos manuales cualificados y en las madres las labores domesticas. Estos autores realizan un análisis de cluster y dendograma para integrar la información sobre la dieta.

Palenzuela y $\mathrm{col}^{8}$ encuentran un consumo deficitario de lácteos (sólo dos terceras partes toman diariamente), pasta, frutas, verduras y hortalizas. El consumo diario de frutas es un 10\% inferior en los niños de 13 años. Hay un significativo consumo de alimentos con "calorías vacías" (comida rápida, dulces, refrescos). Los resultados son parecidos a los que se observan en población adulta española; los adolescentes se alejan de un patrón saludable de alimentación porque disminuyen la ingesta de legumbres, hortalizas y verduras y aumentan la de embutidos, zumos, refrescos. Resultados similares obtuvo el estudio HELENA en una amplia muestra adolescentes europeos. Este estudio refleja que los niños tienen mayores ingestas en general que las niñas excepto de frutas, verduras, postres y derivados lácteos ${ }^{9}$. Además, se asocia la ingesta de bebidas azucaradas y de aperitivos con conductas sedentarias como ver la TV, utilizar el ordenador o jugar con el videojuego ${ }^{10}$. Trabajos recientes en población infanto-juvenil de Navarra encuentran una asociación directa entre la ingesta de refrescos azucarados y el riesgo de obesidad ${ }^{11}$.

Es crucial la puesta en marcha de estrategias de modificación hábitos alimentarios para conseguir mejorar la salud de la población adolescente que será la futura población adulta. Recientemente, nuestro grupo aplicó un programa integral (dieta y actividad física) dirigido a la pérdida de peso en adolescentes de 5 ciudades de España, el programa EVASYON $^{12,13}$. Los hallazgos de nuestro trabajo se relacionan con el perfil genético y epigenético de los sujetos. Así, adolescentes obesos con determinadas variantes genéticas (gen del receptor de melanocortina 4 o FTO (fat mass associated gene) o marcadores epigenéticos tuvieron mejor respuesta al tratamiento ${ }^{14,15}$. Por otro, la pérdida de masa grasa se asoció con una menor velocidad de acortamiento de los telómeros, biomarcadores del envejecimiento celular ${ }^{16}$.

También es de reseñar el estudio HELENA en adolescentes europeos que subraya que las estrategias de modificación de los hábitos alimentarios se han de dirigir a mejorar la calidad de la dieta y aumen- 
tar el nivel de ejercicio físico ${ }^{9}$. Por otro lado, las diferencias entre sexos queda reflejada en el estudio PLENUFAR4 ${ }^{17}$. Para lograr estas acciones se deben implicar las familias, los educadores, los profesionales sanitarios, los políticos, las instituciones y todo el entramado social y comunitario que hay alrededor de los jóvenes y sus familias.

En resumen, es urgente frenar el avance de la obesidad mediante la adquisición de hábitos de vida saludable (alimentación, actividad física) en edades tempranas para evitar la obesidad y otras enfermedades.

\section{BIBLIOGRAFÍA}

1. Pérez Rodrigo C. Current mapping of obesity. Nutr Hosp 2013; 28 (Suppl 5): 21-31.

2. Schröder H, Ribas L, Koebnick C, Funtikova A, Gomez SF, Fíto M et al. Prevalence of abdominal obesity in spanish children and adolescents. Do we need waist circumference measurements in pediatric practice? PLoS One 2014; 27: 9(1): e87549.

3. Flynn MA, Mcneil DA, Maloff B, Mutasingwa D, Wu M, Ford C et al. Reducing obesity and related chronic disease risk in children and youth: a synthesis of evidence with 'best practice' recommendations. Obes Rev 2006; 7 (Suppl 1): $7-66$.

4. Ochoa MC, Moreno-Aliaga MJ, Martínez-González Ma, Martínez JA, Marti A; Genol MEMBERS. Predictor factors for childhood obesity in a Spanish case-control study. Nutrition 2007; 23: 379-384.

5. MuÑoz M, MaRTi A. Dieta durante la infancia y la adolescencia (Cap. 8). En: Salas- Salvadó J, Bonada-Sanjaume A, Trallero Casañas R, Saló-Solá ME, editores. Nutrición y Dietética Clínica. Barcelona: Masson; 2000. pp. 83-98.

6. Marti A, Muñoz M. La alimentación del niño y el adolescente. En: Muñoz M, García Jalon I, Aranceta J, editores. Nutrición Aplicada y Dietoterapia. $2^{\mathrm{a}}$ ed. Pamplona: Eunsa; 2004. pp. 947-978.

7. Moreno LA, Ochoa MC, Wärnberg J, Marti A, Martínez JA, Marcos A. Treatment of obesity in children and adolescents. How nutrition can work? Int J Pediatr Obes 2008; 3 (Suppl 1): 72-77.

8. Palenzuela Paniagua SM, Pérez Milena A, Pérula de Torres LA, Fernández García Ja, Maldonado Alconada J. La alimentación en el adolescente. An Sist Sanit Navar 2014; 37: 47-58.

9. Jiménez-Pavón D, Sesé Ma, Huybrechts I, Cuenca-García M, Palacios G, Ruiz JR et al. Dietary and lifestyle quality indices with/without physical activity and markers of insulin resistance in European adolescents: the HELENA study. Br J Nutr 2013; 110: 1919-1925.

10. Santaliestra-Pasías AM1, Mouratidou T, Verbestel V, Huybrechts I, Gottrand F, LE DonNe $C$ et al. Food consumption and screen-based sedentary behaviors in European adolescents: the HELENA study. Arch Pediatr Adolesc Med 2012; 166: $1010-1020$.

11. Martín-Calvo N, Martínez-González Ma, Bes-Rastrollo M, Gea A, Ochoa MC, Marti A; Genor Members. Sugar-sweetened carbonated beverage consumption and childhood/adolescent obesity: a case-control study. Public Health Nutr 2014, 31: 1-9.

12. Marqués M, Moleres A, Rendo-Urteaga T, Gómez-Martínez S, Zapatera B, Romero $P$ et al. Design of the nutritional therapy for overweight and obese Spanish adolescents conducted by registered dieticians: the EVASYON study. Nutr Hosp 2012; 27: 165-176. 
13. Martínez-Gómez D, Gómez-Martínez S, Puertollano MA, Nova E, Wärnberg J, VeiGA OL et al. Design and evaluation of a treatment programme for Spanish adolescents with overweight and obesity. The EVASYON Study. BMC Public Health 2009; 9:41.

14. Moleres A, Rendo-Urteaga T, Zulet MA, Marcos A, Campoy C, Garagorri JM et al. Obesity susceptibility loci on body mass index and weight loss in Spanish adolescents after a lifestyle intervention. J Pediatr 2012; 161: 466-470.

15. Moleres A, Campión J, Milagro Fi, Marcos A, Campoy C, Garagorri JM et al. Differential DNA methylation patterns between high and low responders to a weight loss intervention in overweight or obese adolescents: the EVASYON study. FASEB J 2013; 27: 2504-2512.

16. García-Calzón S, Moleres A, Marcos A, Campoy C, Moreno La, Azcona-Sanjulín $\mathrm{MC}$ et al. Telomere length as a biomarker for adiposity changes after a multidisciplinary intervention in overweight/obese adolescents: The EVASYON Study. PLoS One 2014; 9(2): e89828.

17. Santiago S, Zazpe I, Masti A, Cuervo M, Martínez JA, Gender differences in lifestyle determinants of overweight prevalence in a sample of Southern European children. Obes Res Clin Pract 2013; 7: 391-400. 